\title{
Comparison of Different Contrast Agents in Detecting Cardiac Right-to-Left Shunt in Patients with a Patent Foramen Ovale during Contrast-Transthoracic Echocardiography
}

\author{
Enfa Zhao, ${ }^{1}$ Gesheng Cheng, ${ }^{1}$ Yushun Zhang, ${ }^{1}$ Yang Li, ${ }^{2}$ and Yingli Wang ${ }^{2}$ \\ ${ }^{1}$ Department of Structural Heart Disease, The First Affiliated Hospital of Xi'an Jiaotong University, Xian 710061, China \\ ${ }^{2}$ Department of Cardiology, Xianyang Hospital of Yanian University, Xianyang 712000, China \\ Correspondence should be addressed to Yushun Zhang; zys2889@163.com
}

Received 12 August 2017; Accepted 8 November 2017; Published 3 December 2017

Academic Editor: Ramazan Akdemir

Copyright (c) 2017 Enfa Zhao et al. This is an open access article distributed under the Creative Commons Attribution License, which permits unrestricted use, distribution, and reproduction in any medium, provided the original work is properly cited.

\begin{abstract}
The aim of this study is to evaluate the ability of two different contrast agents to detect cardiac right-to-left shunting in patients with a patent foramen ovale during contrast transthoracic echocardiography and transesophageal echocardiography. Eighty-four patients who had migraines or experienced cryptogenic stroke were prospectively enrolled. Contrast echocardiography of the right portion of the heart was performed using an injection of either (i) $8 \mathrm{ml}$ of agitated saline, $1 \mathrm{ml}$ of blood, and $1 \mathrm{ml}$ of air (ASB) or (ii) $4 \mathrm{ml}$ of vitamin $B_{6}$ and $6 \mathrm{ml}$ of sodium bicarbonate solution (VSBS). All patients underwent contrast echocardiography with different contrast agents successively before undergoing transesophageal echocardiography. The diagnostic sensitivity of VSBS and ASB for cardiac shunting diagnosis was $94.23 \%$ and $78.85 \%$, respectively. The diagnostic sensitivity in the VSBS group was significantly higher than that in the ASB group $\left(\chi^{2}=5.283, P=0.022\right)$. The observed semiquantitative shunt grading suggests that the positive rate in the VSBS group was higher than that in the ASB group $(Z=-1.998, P=0.046)$. The use of vitamin $B_{6}$ and sodium bicarbonate solution as a TTE contrast agent yielded a high sensitivity compared with ASB. However, further trials with large sample size are required to confirm this finding.
\end{abstract}

\section{Introduction}

A patent foramen ovale (PFO) is a remnant of normal fetal circulation that remains open after birth and is present in approximately $25 \%$ of adults [1]. PFO has long been considered to be of no clinical significance; however, in recent years, numerous studies have suggested that PFO-right-toleft shunting (RLS) is related to a wide array of disease processes, such as migraine headaches, transient ischemic attack (TIA), cryptogenic stroke, decompression sickness, and platypnea-orthodeoxia syndrome [2-4]. Therefore, it is important to identify PFO-RLS and to determine the degree of shunting severity. PFO-RLS can be detected using three different modalities: contrast-transthoracic echocardiography (c-TTE), contrast-transcranial Doppler (c-TCD), or contrast-transesophageal echocardiography (c-TEE). TEE is currently considered to be the "gold standard" for the diagnosis of PFO. However, technical limitations associated with the procedures are unavoidable, including patients intolerance of the TEE probe or an inability to perform the standard Valsalva maneuver due to the presence of the endoscope in the esophagus under local anesthesia [5]. Additionally, TEE was designed to be semi-invasive, which resulted in discomfort and stress for patients during the examination. These barriers to use have widely reduced the application of TEE in clinical practice. A previous study reported that $\mathrm{c}-\mathrm{TCD}$ has a high sensitivity and a favorable specificity in detecting PFO-RLS when compared to TEE [6]. Unfortunately, c-TCD is unable to differentiate cardiac from pulmonary right-toleft shunts and provides no data on the shape and size of the defect [7]. However, previous study concluded that contrast TCD has a sensitivity and specificity of $97 \%$ and $98 \%$, respectively, compared to contrast TEE as the reference [8]. Furthermore, color flow imaging (CFI) using TTE was shown to be an insensitive (28\%) technique for detecting PFO-RLS [9]. Currently, the detection and semiquantitative assessment 


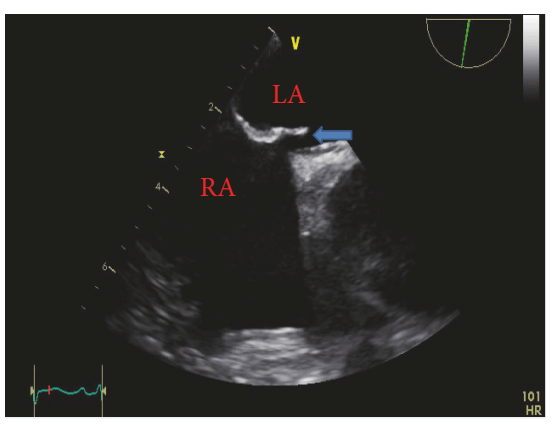

(a)

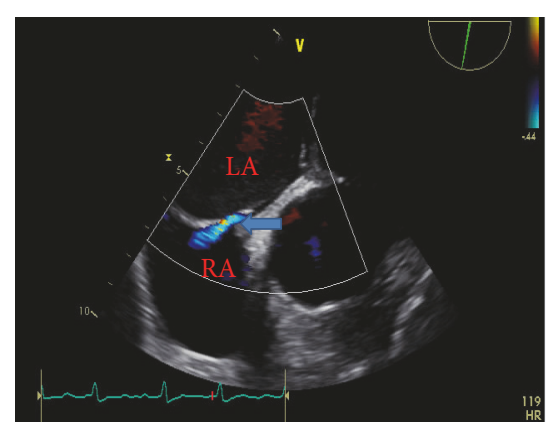

(b)

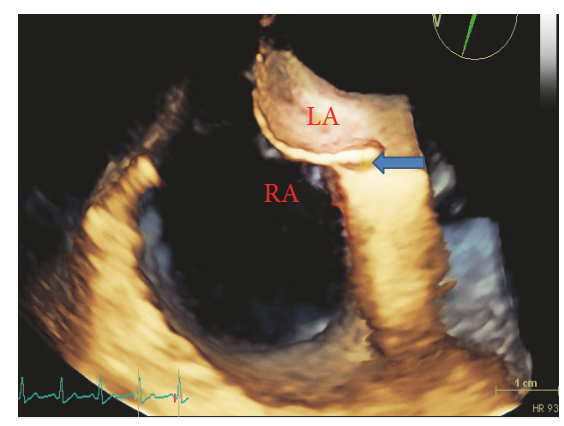

(c)

Figure 1: Transesophageal echocardiography demonstrating the interatrial septum. (a) Two-dimensional transesophageal echocardiography showing a "slit-like" communication between the left and right atria, which was diagnosed as a PFO. (b) Color flow mapping of the spontaneous PFO left-to-right shunt. (c) Detection of PFO by real-time three-dimensional transesophageal echocardiography. The tunnellike configuration between the septum primum and the septum secundum, which indicates the presence of a PFO (blue arrow). LA = left atrium; RA = right atrium.

of PFO-RLS mainly rely on c-TTE. The contrast agents that are currently widely used for the diagnosis of RLS include agitated saline (AS) either alone or with an autologous blood mixture (ASB) [10-13]. Using the acoustic properties of airfilled microbubbles, the contrast agents detect and diagnose PFO-RLS. However, the preparation and injection processes involved in this process are complex and tedious. Vitamin $\mathrm{B}_{6}$ and sodium bicarbonate solution as a c-TTE right heart contrast agent has also been used in clinical practice without noticeable side effects $[14,15]$. The aim of our study was to determine whether the use of a vitamin $\mathrm{B}_{6}$ and sodium bicarbonate solution as a contrast agent results in improved detection of PFO-RLS during c-TTE compared with ASB. Furthermore, we compared the diagnostic sensitivity of VSBS and ASB for cardiac RLS diagnosis with that of TEE, the gold standard.

\section{Methods}

2.1. Patient Population. We performed a prospective study that included 84 patients ( 40 men and 44 women, mean age $41 \pm 16$ [18-65] years) who were highly suspected of having a PFO. Patients who were referred to our echocardiography laboratory after an episode of TIA, cryptogenic stroke, unexplained cerebral infarction, or migraine headache, between 15 November 2014 and 15 March 2015, were enrolled. The study was approved by the Institutional Clinical Ethics Committee of the First Affiliated Hospital of Xi'an Jiaotong University and was performed in accordance with the CONSORT 2010 guidelines and in accordance with the Declaration of Helsinki (1964). All patients or their relatives provided written informed consent. Previous potential intracranial or extracranial, cardiac and extracardiac malformations and lacunar infarction were ruled out using magnetic resonance imaging or computed tomography. Patients who experienced an acute infection period, serious heart and renal insufficiency, atrium fibrillation, serious heart valve disease, blood hypercoagulable state, superior vena cava and right atrial thrombosis, or cognitive dysfunction and those who were unable to undergo the Valsalva maneuver during c-TTE were also excluded.

2.2. TTE Imaging and TEE Examination. The c-TTE bubble study was conducted by an experienced sonographer using the Philips iE33 imaging systems equipped with a S5-1 (1-5 MHz) probe. TEE was performed using the same system fitted with a $2.9-8 \mathrm{MHz}$ multifrequency probe. Half an hour before the TEE procedure, all patients received $2 \%$ lidocaine mucilage for oropharynx anesthesia. The probe was rotated within $45^{\circ}-110^{\circ}$ to clearly display the septum primum and septum secundum as well as to obverse whether an opened PFO and RLS existed both in two-dimensional, threedimensional, and color Doppler ultrasonography (Figure 1). To ensure maximal diagnostic yield, a standard apical fourchamber view was performed with the administration of contrast agents. Gain settings were adjusted to optimize the visualization of the interatrial septum and valvular structures. The ASB contrast agent, including $8 \mathrm{ml}$ of saline solution, $1 \mathrm{ml}$ of air, and $1 \mathrm{ml}$ of autologous blood, was agitated at least 10 times to enhance the backscatter of the ultrasound beam and to achieve good dilution using two $10 \mathrm{ml}$ syringes that were connected by a 3-way stopcock to exchange the air-saline mix [ 9 , $10,13]$. The VSBS contrast agent consisted of $4 \mathrm{ml}$ of vitamin $\mathrm{B}_{6}$ and $6 \mathrm{ml}$ of sodium bicarbonate solution without agitating. The prepared contrast agent was administered intravenously as a bolus via the left antecubital vein. The Valsalva maneuver can enhance the sensitivity of the detection of RLS [9]. All the procedures were conducted with the Valsalva maneuver. The order in which contrast agents were used (ASB or VSBS) was randomly selected for each patient. Furthermore, each contrast material was administered in 30 min intervals. All procedures involved the capture of 10 consecutive beats. The recordings were analyzed retrospectively by two experienced sonographers who were blinded to clinical histories independently. Each bubble study was performed 3 times and the maximum number of microbubbles that was detected was used. The semiquantitative grading of PFO-RLS was classified according to the maximum number of microbubbles that 
TABLE 1: Baseline characteristics of the study population.

\begin{tabular}{lc}
\hline Clinical characteristics & $N(\%)$ \\
\hline Sex (male/female) & $36 / 48(57.0 \%)$ \\
Age $(y)$, mean \pm SD & $39.2 \pm 4.6$ \\
Diabetic mellitus & $10 / 84(11.9 \%)$ \\
Hyperlipidemia & $8 / 84(9.5 \%)$ \\
Arrhythmia & $2 / 84(2.3 \%)$ \\
Hypertension & $11 / 84(13.1 \%)$ \\
Reason for visit & $24 / 84(28.5 \%)$ \\
Ischemic stroke & $20 / 84(23.8 \%)$ \\
Migraine with aura & $8 / 84(9.5 \%)$ \\
Migraine without aura & $17 / 84(20.2 \%)$ \\
TIA & $15 / 84(17.9 \%)$ \\
Cerebral infarction & \\
\hline
\end{tabular}

TIA $=$ transient ischemic attack.

TABLE 2: Diagnostic results of ASB and VSBS compared with those of TEE during the Valsalva maneuver.

\begin{tabular}{|c|c|c|c|c|c|c|c|}
\hline \multirow{2}{*}{ VSBS } & \multicolumn{2}{|c|}{ TEE } & \multirow{2}{*}{ Total } & \multirow{2}{*}{ ASB } & \multicolumn{2}{|c|}{ TEE } & \multirow{2}{*}{ Total } \\
\hline & Positive & Negative & & & Positive & Negative & \\
\hline Positive & 49 & 5 & 54 & Positive & 41 & 4 & 45 \\
\hline Negative & 3 & 27 & 30 & Negative & 11 & 28 & 39 \\
\hline Total & 52 & 32 & 84 & Total & 52 & 32 & 84 \\
\hline
\end{tabular}

$\mathrm{ASB}=$ agitated saline plus blood; $\mathrm{VSBS}=$ vitamin $\mathrm{B}_{6}$ and $6 \mathrm{ml}$ sodium bicarbonate solution; $\mathrm{TEE}=$ transesophageal echocardiography.

appeared in the left atrium on a still frame based on previous criteria $[13,16]$ and was defined according to the following criteria: when no, 1-10 bubbles, 11-30 bubbles, and $>30$ bubbles (or left atrial opacity) were detected, the RLS was considered to be negative, mild, moderate, and extensive, respectively (Figure 2). Cardiac cycle microbubbles appeared in the left atrium after complete opacification of the right atrium was observed. The origin of RLS was determined according to the time by which left atrial microbubbles appeared. That is, for a developing time between 3 and 5 cardiac cycles, RLS was considered to be the result of a PFO, while, for a developing time of more than 5 cardiac cycles, RLS was considered to be the result of pulmonary arteriovenous malformation [17].

2.3. Statistical Analysis. Continuous variables are expressed as the mean \pm standard deviation, and categorical variables are reported as counts and percentages. A chi-square test was used to compare the total positive rates between groups. The Mann-Whitney-Wilcoxon test was used to compare semiquantitative shunt grading using two contrast agents. Statistical significance was assumed when the $P$ value $<0.05$. All data were analyzed using SPSS software (version 18.0.1, SPSS Inc.).

\section{Results}

3.1. Baseline Characteristics and Positive Detection Rate of Different Contrast Agents. The baseline characteristics of the population included in this study are shown in Table 1. Among the 84 patients who were given different contrast agents, no patient reported a noticeable adverse event during the procedure or during a 12-hour follow-up period. In total, 52 patients were diagnosed with a PFO. Regardless of the degree of shunting severity, the diagnostic sensitivity of VSBS and ASB was $94.23 \%$ and $78.85 \%$, respectively. VSBS yielded a higher sensitivity than did ASB $\left(\chi^{2}=5.283, P=0.022\right)$ (Table 2). However, there was no statistical difference with respect to specificity between the VSBS and ASB groups (84.38\% versus $87.50 \%, P>0.05)$. ASB exhibited high concordance with TEE for cardiac RLS diagnosis $(\kappa=0.637)$. VSBS exhibited higher concordance with TEE for cardiac RLS diagnosis $(\kappa=0.796)$.

3.2. Semiquantitative Shunt Grading. According to the semiquantitative grading of PFO-RLS, the degree of PFO-RLS was categorized into four grades. The specific results of the semiquantitative shunt grading are provided in Table 3 . The semiquantitative grading of two different contrast agents indicated that the RLS positive rate in the VSBS group was higher than that in the ASB group $(Z=-1.998, P=0.046)$.

\section{Discussion}

To our knowledge, this is the first prospective observational study in which vitamin $\mathrm{B}_{6}$ and sodium bicarbonate solution was shown to be a better contrast agent than ASB for detecting PFO-RLS. Our preliminary study indicated that using a vitamin $\mathrm{B}_{6}$ and sodium bicarbonate solution as a c-TTE contrast agent yielded a higher sensitivity than that with ASB, without producing noticeable adverse events compared to 
TABLE 3: Results of the semiquantitative shunt grading using ASB and VSBS during the Valsalva maneuver.

\begin{tabular}{lcc}
\hline & ASB $(n=84)$ & VSBS $(n=84)$ \\
\hline Negative & 39 & 30 \\
Positive & 45 & 54 \\
Mild & 17 & 11 \\
Moderate & 18 & 27 \\
Extensive & 10 & 16 \\
\hline
\end{tabular}

$\mathrm{ASB}=$ agitated saline plus blood; VSBS $=$ vitamin $\mathrm{B}_{6}$ and $6 \mathrm{ml}$ sodium bicarbonate solution.

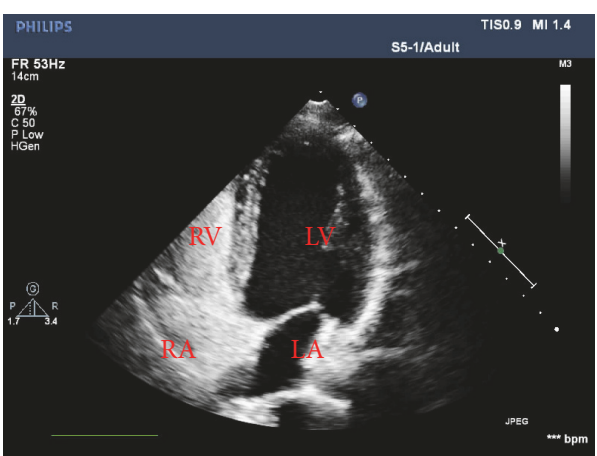

(a)

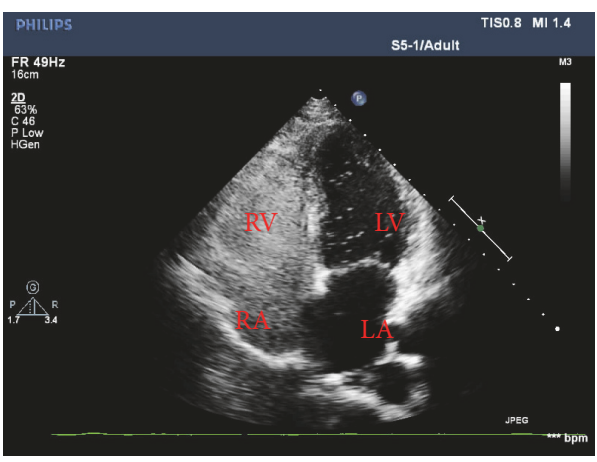

(c)

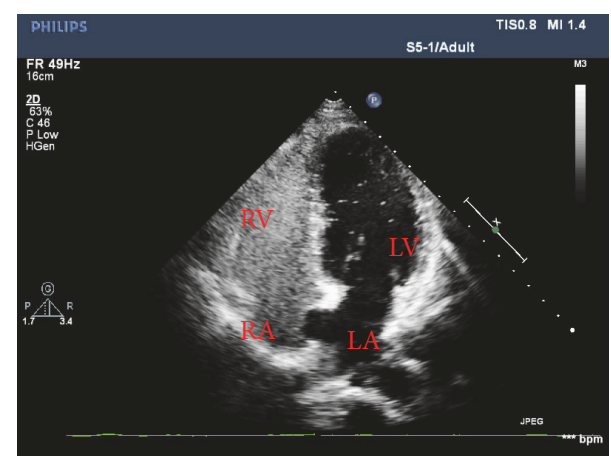

(b)

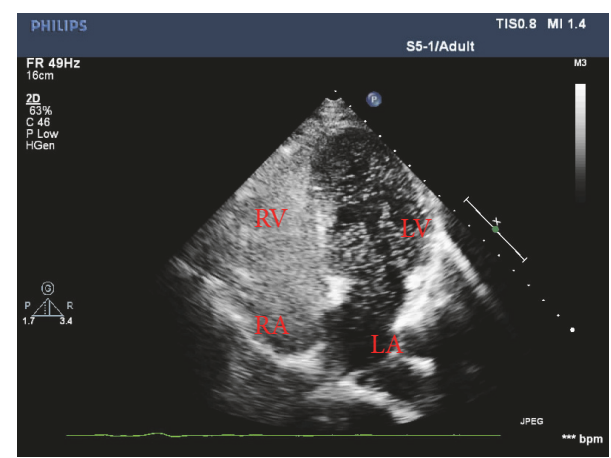

(d)

FIGURE 2: Semiquantitative grading of a patent foramen ovale-right-to-left shunt by transthoracic echocardiography using the bubble test. (a) No right-to-left shunt. (b) Mild right-to-left shunt (1-10 microbubbles in the left atrium). (c) Moderate right-to-left shunt (11-30 microbubbles in the left atrium). (d) Extensive right-to-left shunt (more than 30 microbubbles in the left atrium or left atrial opacity). LA = left atrium; RA = right atrium; $L V=$ left ventricle; $R V=$ right ventricle.

that observed with TEE, which was used as the reference. It has previously been reported that the diagnostic sensitivity of TTE compared to that of TEE (when used as the reference) ranged from 23\% (95\% confidence interval [CI]: 11\%-38\%) to $92 \%$ (95\% CI: $62 \%-100 \%)$ [18-23] which reflects the use of different contrast agents, different microbubble cutoffs for a positive TTE/TEE, and different cardiac cycle cutoffs for a positive TTE/TEE. In our study, we measured a sensitivity of $94.23 \%$ and $78.85 \%$ for VSBS and ASB, respectively, which was consistent with previous studies. A recent study showed that the sensitivity and specificity of VSBS were $92.31 \%$ and $84.88 \%$, respectively, when TEE was used as the reference. Additionally, in our study, we found no difference with respect to specificity between the VSBS and ASB groups (84.38\% versus $87.50 \%, P>0.05)$.
Determining whether PFO is clinically significant and merits treatment is determined by the presence of PFORLS and the degree of shunt severity [15]. Currently, the detection of PFO-RLS mainly relies on C-TTE. However, the type of contrast agent has a great influence on the detection of PFO-RLS and the degree of shunting [24]. The most widely used contrast agents currently include AS or ASB [25]. It has generally been believed that adding blood to AS can stabilize microbubbles and increase their suspension time in the blood [24]. Similarly, agitated saline with blood can also emulsify the microbubbles and prevent them from dissolving in systemic circulation [12], which facilitates the passage of more microbubbles through the PFO. Meanwhile, mixing blood with agitated saline can result in an increase in the viscosity of the contrast material and can further reduce the 
rate of injection. Whether the presence of air microbubbles in the blood causes adverse effects remains controversial. Indeed, improper operation will result in large microbubbles and blood clots [26]. However, the contrast agents that are commonly used are heterogeneously mixed. Notably, this requires a complex and cumbersome agent injection, which involves two $10 \mathrm{ml}$ syringes connected by a 3-way stopcock to exchange the air-saline mix [27]. A vitamin $\mathrm{B}_{6}$ and sodium bicarbonate solution is convenient to obtain for clinical use. VSBS is a safe and effective contrast agent used for c-TTE that has little side effect. Furthermore, it is applied to the diagnosis of congenital heart disease $[14,15]$. Vitamin $\mathrm{B}_{6}$ and sodium bicarbonate interact in solution in a traditional acid-base neutralization reaction, which does not result in changes to the structure of vitamin $\mathrm{B}_{6}$. The neutralization reaction occurs between the hydrochloride group provided by vitamin $B_{6}$ and bicarbonate when the two chemicals are mixed [15]. When VSBS, which is an affordable agent, was injected, the reaction product is carbon dioxide, which is characteristically safe. VSBS contrast agent does not require a three-way stopcock and exchange to avoid blood splashing that is otherwise caused by an improper exchanging operation. VSBS also avoids the distressing patients, who otherwise are concerned about injection of visible gas into the body. However, the neutralization reaction between vitamin $B_{6}$ and bicarbonate produces more carbon dioxide and a longer microbubbles' peak time, which improves the opportunity to observe and diagnose PFO-RLS [15]. Among a variety of gases that could be used during the right heart contrast echocardiography, carbon dioxide has a superior solubility and diffusion rate. Therefore, carbon dioxide has been found to be the safest for use in the blood [28]. In our study, no patient reported an obvious adverse event during the procedure or during the 12-hour follow-up. Therefore, it is expected that the procedure described herein is the preferred contrast agent for the diagnosis of PFO-RLS.

This preliminary study has several limitations. Poor intolerance among patients for the TEE probe and failure to perform a standard Valsalva maneuver while the TEE was being performed may affect the accuracy of the results to some extent. Another limitation is the small sample size of our study. Further prospective multicenter studies with larger populations are warranted to confirm and extend the conclusions of our study.

Our preliminary study indicated that the application of vitamin $B_{6}$ and sodium bicarbonate solution as a c-TTE contrast agent yielded a higher sensitivity compared to ASB without noticeable adverse events when TEE was used as the reference. Therefore, vitamin $\mathrm{B}_{6}$ and sodium bicarbonate solution may be used during contrast-transthoracic echocardiography for the diagnosis of PFO-RLS giving its simplicity, feasibility, and noninvasive nature.

\section{Conflicts of Interest}

The authors have declared that no conflicts of interest exist.

\section{Authors' Contributions}

Enfa Zhao, Gesheng Cheng, and Yang Li wrote the paper. Yingli Wang and Yushun Zhang participated in the discussion of the manuscript. All authors read and approved the final manuscript.

\section{Acknowledgments}

This work was supported by Shaanxi Provincial Science and Technology Integrated Innovation Project plan (no. 2014KTCL03-11). The authors thank the staff of the First Affiliated Hospital of Xian Jiaotong University for their support in patient care and research.

\section{References}

[1] A. Drighil, H. El Mosalami, N. Elbadaoui, S. Chraibi, and A. Bennis, "Patent foramen ovale: A new disease?" International Journal of Cardiology, vol. 122, no. 1, pp. 1-9, 2007.

[2] E. K. Kerut, W. T. Norfleet, G. D. Plotnick, and T. D. Giles, "Patent foramen ovale: A review of associated conditions and the impact of physiological size," Journal of the American College of Cardiology, vol. 38, no. 3, pp. 613-623, 2001.

[3] H. Hara, R. Virmani, E. Ladich et al., "Patent foramen ovale: current pathology, pathophysiology, and clinical status," Journal of the American College of Cardiology, vol. 46, no. 9, pp. 17681776, 2005.

[4] M. K. Mojadidi, R. Gevorgyan, N. Noureddin, and J. M. Tobis, "The effect of patent foramen ovale closure in patients with platypnea-orthodeoxia syndrome," Catheterization and Cardiovascular Interventions, vol. 86, no. 4, pp. 701-707, 2015.

[5] G. Souteyrand, P. Motreff, J.-R. Lusson et al., "Comparison of transthoracic echocardiography using second harmonic imaging, transcranial Doppler and transesophageal echocardiography for the detection of patent foramen ovale in stroke patients," European Heart Journal - Cardiovascular Imaging, vol. 7, no. 2, pp. 147-154, 2006.

[6] L. Caputi, M. R. Carriero, C. Falcone et al., "Transcranial Doppler and Transesophageal Echocardiography: Comparison of Both Techniques and Prospective Clinical Relevance of Transcranial Doppler in Patent Foramen Ovale Detection," Journal of Stroke and Cerebrovascular Diseases, vol. 18, no. 5, pp. 343348, 2009.

[7] M. Komar, M. Olszowska, T. Przewłocki et al., "Transcranial doppler ultrasonography should it be the first choice for persistent foramen ovale screening?" Cardiovascular Ultrasound, vol. 12, no. 1, article no. 16, 2014.

[8] T. González-Alujas, A. Evangelista, E. Santamarina et al., "Diagnosis and quantification of patent foramen ovale. which is the reference technique? simultaneous study with transcranial doppler, transthoracic and transesophageal echocardiography," Revista Española de Cardiología, vol. 64, no. 2, pp. 133-139, 2011.

[9] K. Marriott, V. Manins, A. Forshaw, J. Wright, and R. Pascoe, "Detection of right-to-left atrial communication using agitated saline contrast imaging: Experience with 1162 patients and recommendations for echocardiography," Journal of the American Society of Echocardiography, vol. 26, no. 1, pp. 96-102, 2013.

[10] S. Thanigaraj, A. Valika, A. Zajarias, J. M. Lasala, and J. E. Perez, "Comparison of Transthoracic Versus Transesophageal Echocardiography for Detection of Right-to-Left Atrial Shunting Using Agitated Saline Contrast," American Journal of Cardiology, vol. 96, no. 7, pp. 1007-1010, 2005.

[11] M. C. Lange, V. F. Zétola, E. J. Piovesan, and L. C. Werneck, "Valsalva maneuver procedures in the diagnosis of right-to-left 
shunt by contrast-enhanced transcranial doppler using agitated saline solution with blood as a contrast agent," Arquivos de Neuro-Psiquiatria, vol. 68, no. 3, pp. 410-413, 2010.

[12] A. Shariat, E. Yaghoubi, R. Nemati, K. Aghasadeghi, and A. B. Haghighi, "Comparison of agitated saline mixed with blood to agitated saline alone in detecting right-to-left shunt during contrast-transcranial Doppler sonography examination," Acta Neurologica Taiwanica, vol. 20, no. 3, pp. 182-187, 2011.

[13] E. Zhao, Y. Wei, Y. Zhang, N. Zhai, P. Zhao, and B. Liu, "A comparison of transthroracic echocardiograpy and transcranial Doppler with contrast agent for detection of patent foramen ovale with or without the Valsalva maneuver," Medicine (United States), vol. 94, no. 43, Article ID e1937, 2015.

[14] Q. Li, X. Yu, and Y. Xiong, "A New Type of Right Heart Ultrasonic Contrast Agent," Chinese Journal of Ultrasound in Medicine, vol. 15, no. 9, pp. 672-674, 1999.

[15] J.-C. He, J.-Y. Zheng, X. Li et al., "Transthoracic contrast echocardiography using vitamin B6 and sodium bicarbonate as contrast agents for the diagnosis of patent foramen ovale," The International Journal of Cardiovascular Imaging, pp. 1-7, 2017.

[16] H. W. Schuchlenz, W. Weihs, S. Horner, and F. Quehenberger, "The association between the diameter of a patent foramen ovale and the risk of embolic cerebrovascular events," American Journal of Medicine, vol. 109, no. 6, pp. 456-462, 2000.

[17] K. Zukotynski, R. P. Chan, C.-M. Chow, J. H. Cohen, and M. E. Faughnan, "Contrast echocardiography grading predicts pulmonary arteriovenous malformations on CT," CHEST, vol. 132, no. 1, pp. 18-23, 2007.

[18] J.-W. Ha, M.-S. Shin, S. Kang et al., "Enhanced detection of right-to-left shunt through patent foramen ovale by transthoracic contrast echocardiography using harmonic imaging," American Journal of Cardiology, vol. 87, no. 5, pp. 669-671, 2001.

[19] H. W. Rahmouni, M. G. Keane, F. E. Silvestry et al., "Failure of digital echocardiography to accurately diagnose intracardiac shunts," American Heart Journal, vol. 155, no. 1, pp. 161-165, 2008.

[20] C. Zito, G. Dattilo, G. Oreto et al., "Patent foramen ovale: Comparison among diagnostic strategies in cryptogenic stroke and migraine," Journal of Echocardiography, vol. 26, no. 5, pp. 495503, 2009.

[21] H. P. Kühl, R. Hoffmann, M. W. Merx et al., "Transthoracic echocardiography using second harmonic imaging: Diagnostic alternative to transesophageal echocardiography for the detection of atrial right to left shunt in patients with cerebral embolic events," Journal of the American College of Cardiology, vol. 34, no. 6, pp. 1823-1830, 1999.

[22] I. Monte, S. Grasso, S. Licciardi, and L. P. Badano, "Head-tohead comparison of real-time three-dimensional transthoracic echocardiography with transthoracic and transesophageal twodimensional contrast echocardiography for the detection of patent foramen ovale," European Heart Journal - Cardiovascular Imaging, vol. 11, no. 3, pp. 245-249, 2010.

[23] R. Stendel, H.-J. Gramm, K. Schröder, C. Lober, and M. Brock, "Transcranial doppler ultrasonography as a screening technique for detection of a patent foramen ovale before surgery in the sitting position," Anesthesiology, vol. 93, no. 4, pp. 971-975, 2000.

[24] M. Gentile, A. De Vito, C. Azzini, C. Tamborino, and I. Casetta, "Adding Blood to Agitated Saline Significantly Improves Detection of Right-to-Left Shunt by Contrast-Transcranial ColorCoded Duplex Sonography," Ultrasound in Medicine \& Biology, vol. 40, no. 11, pp. 2637-2641, 2014.
[25] T. D. Woods and A. Patel, "A critical review of patent foramen ovale detection using saline contrast echocardiography: when bubbles lie," Journal of the American Society of Echocardiography, vol. 19, no. 2, pp. 215-222, 2006.

[26] D.-S. Jeon, H. Luo, T. Iwami et al., “The usefulness of a 10\% air$10 \%$ blood- $80 \%$ saline mixture for contrast echocardiography: Doppler measurement of pulmonary artery systolic pressure," Journal of the American College of Cardiology, vol. 39, no. 1, pp. 124-129, 2002.

[27] B. E. Fenster, D. Curran-Everett, A. M. Freeman, H. D. Weinberger, J. Kern Buckner, and J. D. Carroll, "Saline contrast echocardiography for the detection of patent foramen ovale in hypoxia: A validation study using intracardiac echocardiography," Journal of Echocardiography, vol. 31, no. 4, pp. 420-427, 2014.

[28] K. M. Christmas and J. B. Bassingthwaighte, "Equations hfor $\mathrm{O}_{2}$ and $\mathrm{CO}_{2}$ solubilities in saline and plasma: combining temperature and density dependences," Journal of Applied Physiology, 1985. 


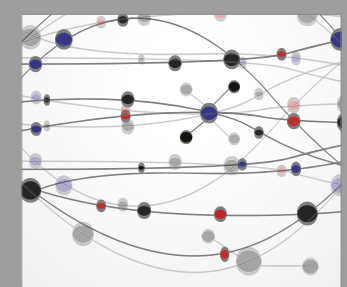

The Scientific World Journal
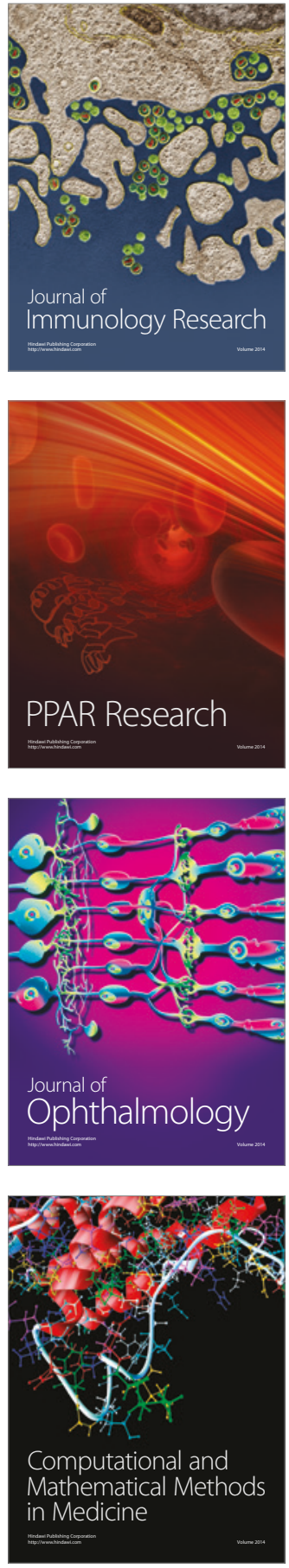

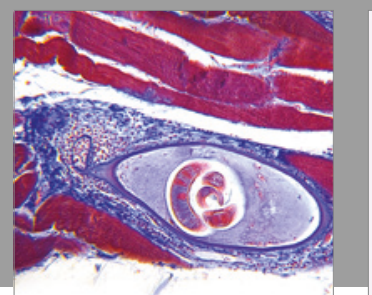

Gastroenterology Research and Practice
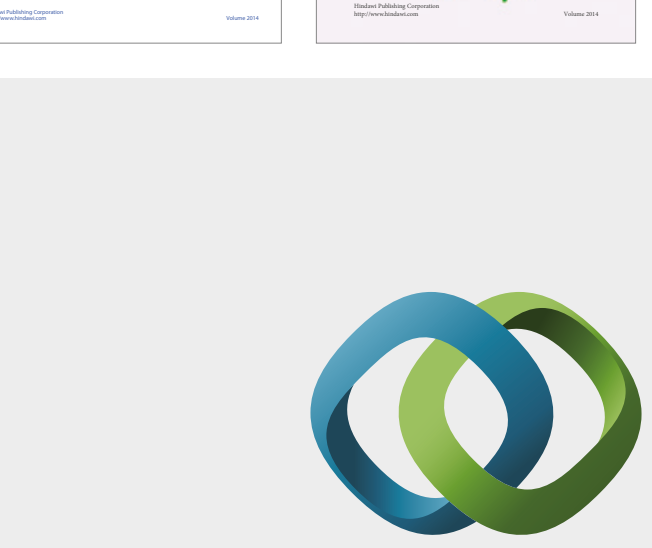

\section{Hindawi}

Submit your manuscripts at

https://www.hindawi.com
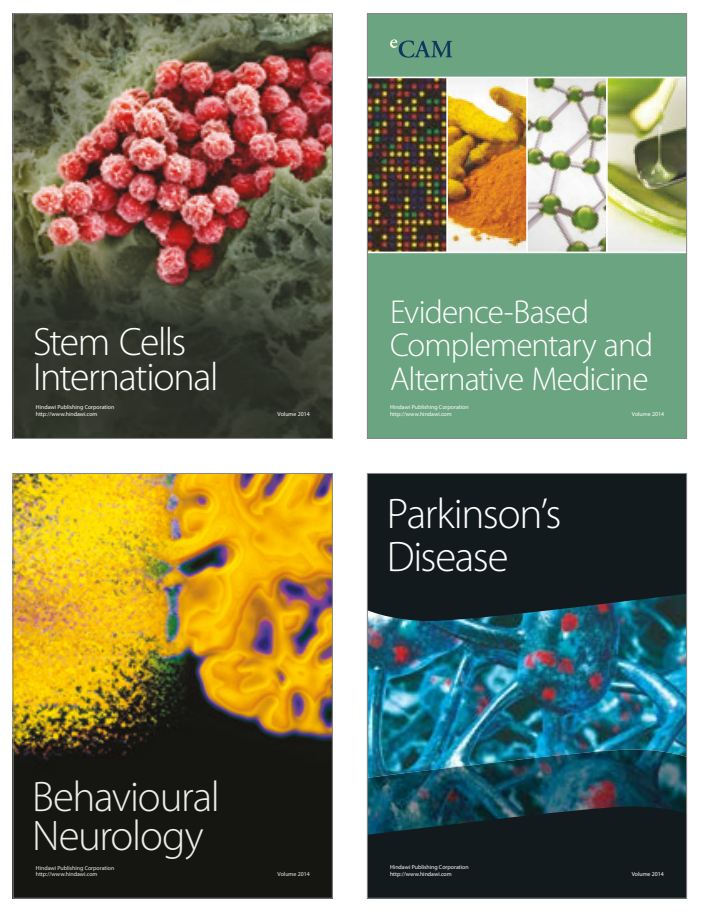
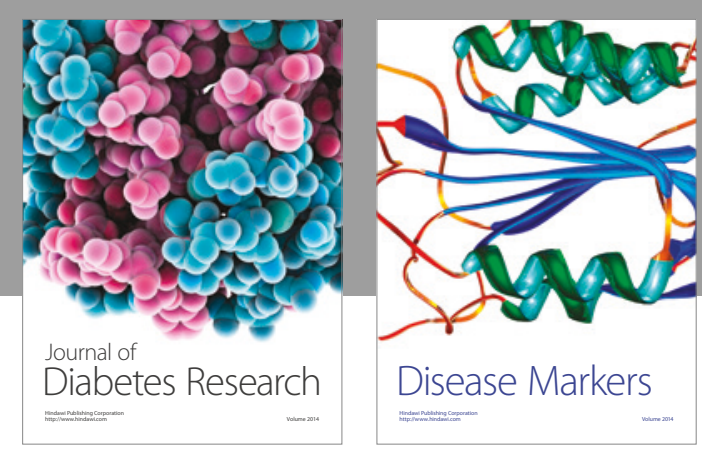

Disease Markers
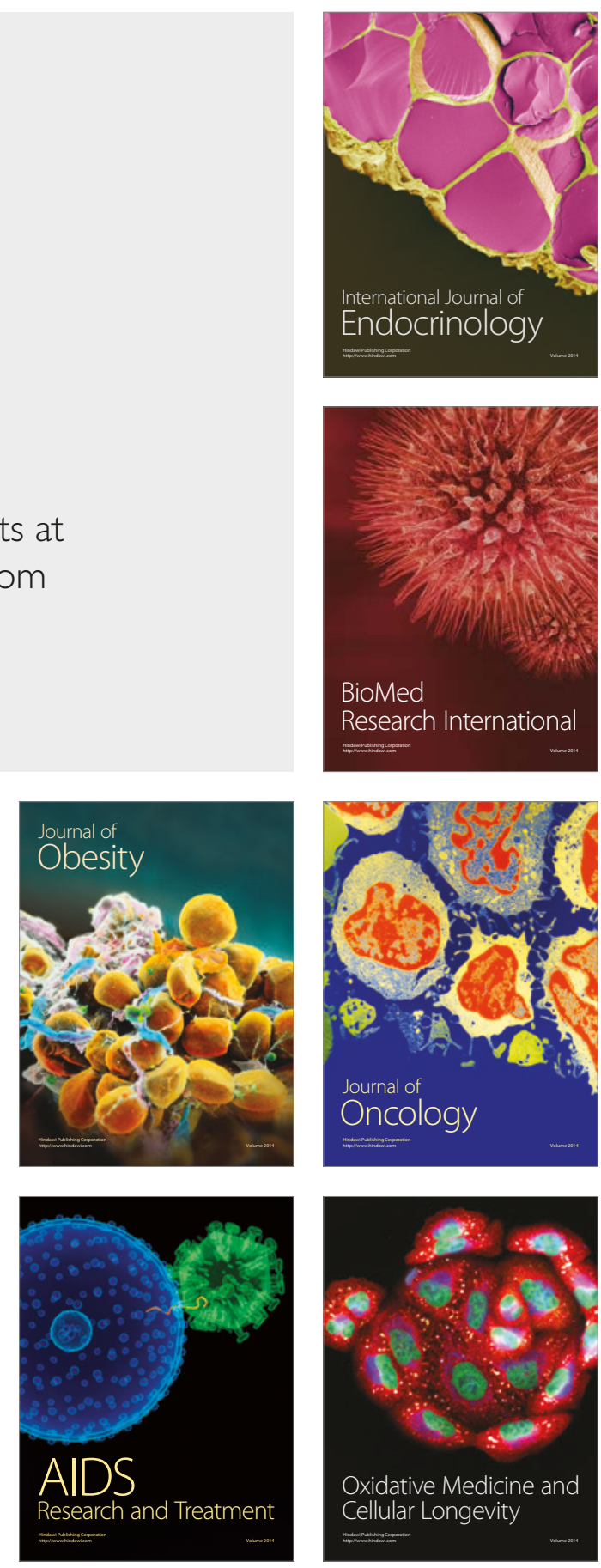